\title{
Transmission in the vicinity of the Dirac point in hexagonal Photonic Crystals
}

\author{
Marcus Diem,, ,* Thomas Koschny, ${ }^{1,2}$ and C. M. Soukoulis ${ }^{1,2}$ \\ ${ }^{1}$ Ames Laboratory and Department of Physics and Astronomy, \\ Iowa State University, Ames, IA, 50011 \\ ${ }^{2}$ Institute of Electronic Structure (IESL) and Laser, \\ Foundation for Research Technology Hellas (FORTH) \\ and Department of Material Science and Technology, \\ University of Crete, 71110 Heraklion, Crete, Greece
}

(Dated: November 1, 2018)

\begin{abstract}
We use a scattering matrix approach to simulate the transmission through a hexagonal Photonic Crystal in the vicinity of the Dirac point. If the crystal is oriented so that the propagation direction perpendicular to the surface corresponds to the $\Gamma \mathrm{K}$ direction, no oblique transmission is possible for a very long (infinite) structure. For a finite structure with width, W, and length, L, the length dependence of the transmission is given by $T_{\text {total }}=\Gamma_{0} \mathrm{~W} / \mathrm{L}$. For $T_{\text {total }}$ all waves with a wavevector parallel to the surface, $k_{\|}=n \frac{2 \pi}{\mathrm{W}}$, described by a channel number, $n$, must be considered. We show the transmission at the Dirac point follows the given scaling law and this scaling law is related to the behavior of the individual channels. This leads to the establishment of a criterion for the maximum length for this scaling behavior when the total transmission reaches a constant value. We also compare this scaling behavior to the results in other frequency regions.
\end{abstract}

PACS numbers: 42.70.Qs,41.20.Jb,42.25.Bs 


\section{INTRODUCTION}

The simulation of two-dimensional Photonic Crystals (PC) with a hexagonal lattice has so far primarily focused on studying the band gap, either to obtain the largest possible $\operatorname{gap}^{1,2,3}, 4, \underline{5}, \underline{6}$ or to study the impact of disorder on the width of the gap $\underline{7,8,9,10}$ or wave-guiding properties in such crystals. $\underline{11}$ The transmission in the band regions was used to characterize experimental samples $\frac{12}{2}$ or for studies of negative refraction. $13,14,15,16,17,18,19$ For a general overview see review by Busch et al. and references therein.$^{20}$

Recently, Raghu and Haldane pointed out that the K-point in the band-structure can also be seen as the optical analogue to the Dirac point in graphene. ${ }^{21,22}$ At these points the band-structure exhibits a conical singularity with a linear dispersion relation, as it occurs in the Dirac equation. This offers the possibility to discuss many interesting effects of this dispersion predicted in the electronic case for graphene, 23,24 such as changes in the conductance fluctuations $^{25}$ and enhanced transmittance in the disordered $\operatorname{case}^{26}, 27$ in a noninteracting photonic system.

Around the Dirac point, a pseudo-diffusive transmission behavior characterized by a scaling of the transmission proportional to $\mathrm{W} / \mathrm{L}$ with the width, $\mathrm{W}$, and length, L, of the PC was predicted. ${ }^{28}$ This result was obtained in an analytic approach by discretizing the incoming modes into channels with a spacing of the wavevector parallel to the surface, $k_{\|}$, by $\Delta k_{\|}=\frac{2 \pi}{\mathrm{W}}$ and by using current conservation and symmetry relation in a transfer matrix approach analog to a calculation for graphene. ${ }^{27,29}$ Numerical studies, using the multiplescattering Korringa-Kohn-Rostocker method ${ }^{30}$ or finite difference time-domain ${ }^{31}$ confirmed the results. However, in both numerical approaches, only short crystals and only a small number of lengths have been studied and the behavior of individual channels has been ignored as well.

In this paper, we investigate the contribution of these channels and show there is also a width-dependent upper length limit for the W/L scaling for long crystals associated with the complete suppression of all channels except the $0^{\text {th }}$ one with $k_{\| 1}=0.0$. This behavior is very important in understanding the predicted enhancement of transmittance at the Dirac point in disordered Photonic Crystals. 28

We use our own implementation of a Fourier-Modal method with a scattering matrix approach, also known as Rigorous-Coupled Wave Analysis (RCWA), 2,33 which allows us to 


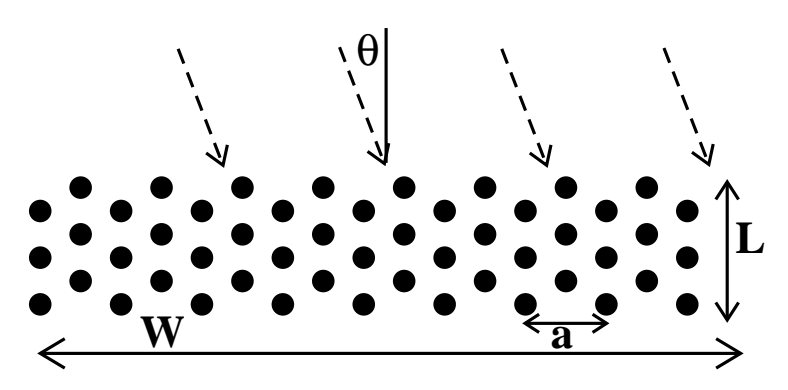

FIG. 1: Structure. The shown example has a length of 3 unit cells and a width of 8 . A plane wave with an angle $\theta=0$ (perpendicular to the surface) propagates along the $\Gamma \mathrm{K}$ direction in the band-structure. One unit cell in the propagation direction contains 2 rows of cylinders.

simulate crystals of arbitrary length, L, and to determine the limit on $W>>L$ not discussed in previous publications. Special care is taken of the correct Fourier-factorization rules to ensure a fast convergence. $\underline{\underline{34}, 35,36}$ This approach assumes incoming plane waves, defined by a dimensionless frequency $\omega^{\prime}=\omega a / 2 \pi c=\mathrm{a} / \lambda$ and the angle $\theta$ to the surface normal (Fig.(1) onto a periodic structure with lattice constant $a$.

The transmittance, $\mathrm{T}$, for one frequency-angle pair through the structure is calculated by summing over all propagating diffraction orders and adding up the magnitude of their Poynting vectors. The same holds for the reflectance, $\mathrm{R}$, and the sum of both is tested to be equal to unity. In the studied case the finite width is incorporated by the superposition of $2 N+1$ planes waves with different $k_{\text {॥ }}$ corresponding to the channels discussed above. Each channel with number $n$ is associated with a $k_{1, n}=n \frac{2 \pi}{\mathrm{W}}$ and an angle to the surface normal given by $\theta=\arcsin \left(\frac{k_{\Perp}}{k_{0}}\right)$ with $k_{0}=2 \pi \omega^{\prime}$. The maximum/minimum parallel component of the wavevector is then given by $\pm N \frac{2 \pi}{\mathrm{W}}$. For crystals with a length $L>1 / k_{1, \max }$, the summed transmission of all channels, $\sum_{n=-N}^{N} \mathrm{~T}_{k_{1, n},}$, is supposed to be independent of $k_{\|, \max }$. For this approach to be valid, a wide and short crystal must be assumed, so that the details of the edges become less important. $\underline{28}$

As a model system, we use cylinders $(r / a=0.225, \epsilon=14.0)$ in air $(\epsilon=1.0)$ on a hexagonal lattice. The crystal orientation is chosen, so a plane wave at perpendicular incidence propagates along the $\Gamma \mathrm{K}$ direction, for which the Dirac point occurs in H-polarization (magnetic field parallel to the cylinders). The corresponding band-structure, together with the transmittance for H-polarization in the $\Gamma \mathrm{K}$ direction corresponding to $\theta=0^{\circ}$, is shown in Fig.2, The Dirac point occurs at $\omega_{D}^{\prime}=0.5294$ in the band-structure. 


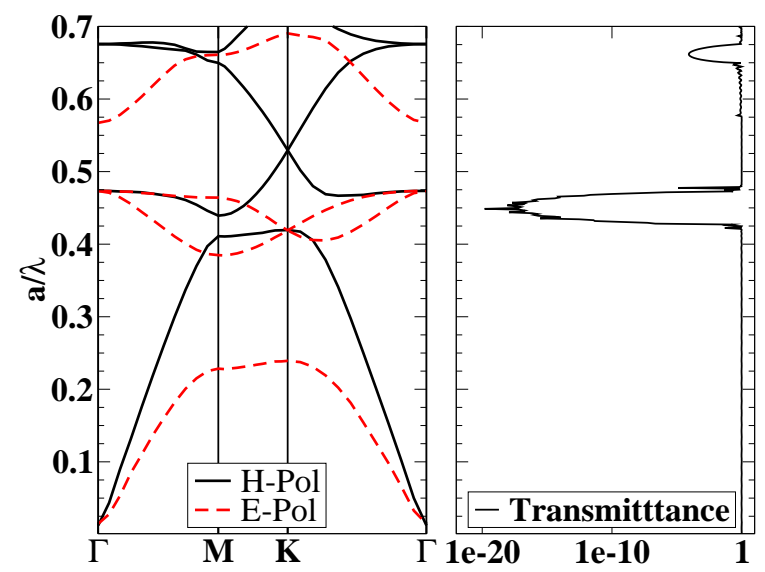

FIG. 2: (Color online) Photonic band structure for dielectric cylinders in air on a hexagonal lattice with $r / a=0.225$ and $\epsilon=14.0$. The arrow marks the Dirac point at $\omega_{D}^{\prime}=0.5294$. For Hpolarization (magnetic field parallel to the cylinders) no propagating modes exist at that frequency except for the one at the Dirac point. On the right side the transmittance for H-polarization in the $\Gamma \mathrm{K}$ direction $\left(\theta=0^{\circ}\right)$ is shown.

The dependence of the transmittance (sum over all propagating diffraction orders) on the angle and frequency of the incident wave is shown in Fig. 3 for a spacing of $\Delta \theta=1^{\circ}$. The features of the band-structure, such as the stop band $\left(\omega^{\prime} \approx 0.45\right)$, and the pseudostop band around $\left(\omega^{\prime} \approx 0.675\right)$ can be identified. Figure $3 \mathrm{~b}$ enlarges the region around the Dirac point for positive and negative angles. From the smallest width of the conical shaped transmittance, the Dirac point can be estimated around $\omega^{\prime} \approx 0.532$. However, resonances, due to the finite size, make a very precise determination more difficult and a better way will be discussed later. In this case higher order diffraction orders do not contribute significantly to the total transmittance. This is in contrast to the reflectance (not shown) where for angles larger than $10-15^{\circ}$ most energy is transferred in the $\pm 1^{\text {st }}$ diffraction order, depending upon whether the angle on the incoming wave is positive $\left(-1^{\text {st }}\right)$ or negative $\left(+1^{\text {st }}\right)$. It should be noted, in these plots individual angles cannot be assigned a channel number, since the spacing is not equidistant in $k_{1}$.

Due to linear dispersion relation around the Dirac point, the phase of a plane wave with perpendicular incidence (in $\Gamma K$-direction) changes linearly with frequency, if phase changes at the surfaces of the crystal are constant for all considered frequencies. The phase change in the transmittance calculation, $\Delta \Phi_{\mathrm{T}}$, in a given frequency interval $\Delta \omega^{\prime}$, is then equal to 

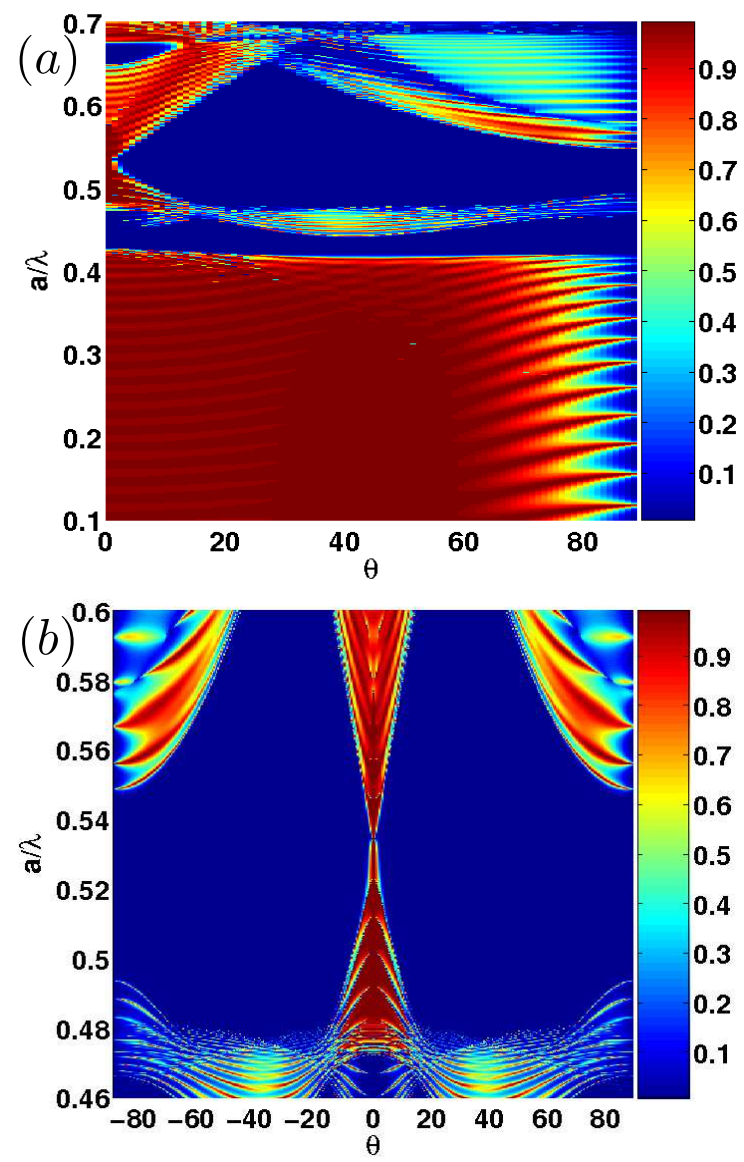

FIG. 3: (Color online) (a) Angular and frequency-dependence of the transmittance (sum over all diffraction orders) through a structure consisting of 40 cylinder rows for H-polarization. $\theta=0^{\circ}$ corresponds to propagation in $\Gamma K$ direction. The result is symmetric in the angle $\theta$. (b) Enlargement around the Dirac point $\left(\omega_{D}^{\prime} \approx 0.532\right)$. The total transmittance, $\mathrm{T}$, discussed later in the paper, corresponds to a summation of the transmittance over all angles with $\theta=\arcsin \left(n k_{\| 1} / k_{0}\right)$ $n=-N, \cdots, N$ (equidistant in $k_{\|}$not in $\theta$ ) for a each frequency.

the product of the length times the change of the wavevector in the band-structure in the same frequency interval $\left(\Delta \Phi_{\mathrm{T}}\left(\Delta \omega^{\prime}\right)=L \Delta k\left(\Delta \omega^{\prime}\right)\right)$. Although not shown, our numerical results exhibit this behavior extremely well. The absolute phase can only be determined up to an arbitrary but constant shift. As will be shown later, a precise determination of the Dirac frequency is essential. However, since the band-structure and transmittance are calculated by different methods, a small difference in the Dirac frequency is found and the exact frequency for the transmittance calculations must be determined within the RCWA method. 


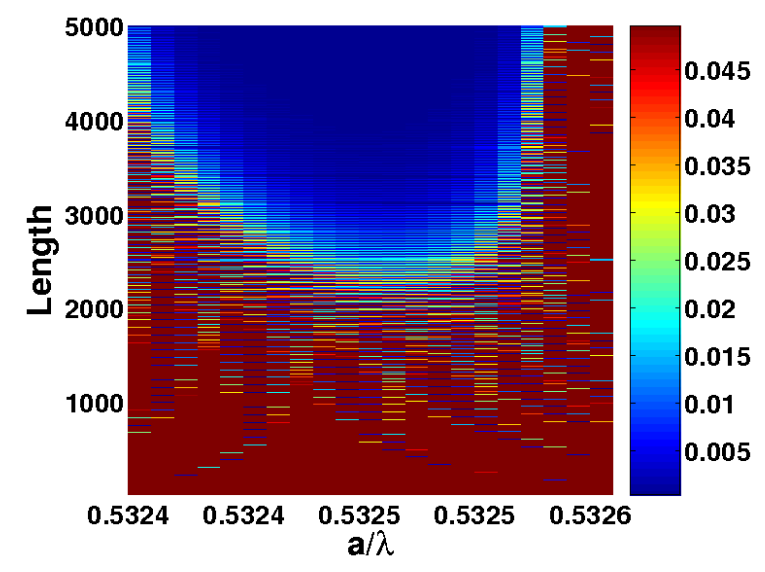

FIG. 4: (Color online) Transmittance of the $1^{\text {st }}$ channel with $k_{\text {।I }}=2 \pi / W=0.0013$ for $\mathrm{W}=5000$ over frequency. At the Dirac point $\left(\omega_{D}^{\prime}=0.5325\right)$ the transmittance is lowest for long structures.

This is possible by looking at the transmittance for a fixed $k_{11}$, preferably close to zero, and choosing the frequency for which this transmittance becomes the smallest for long structures. At the Dirac point only the $k_{11}=0.0$ component propagates in long structures. In Fig. 4 the length-dependent transmittance is plotted for different frequencies for $k_{\| 1}=0.00125$ corresponding to the $1^{\text {st }}$ channel with a width of $\mathrm{W}=5000$. We determine the frequency for the Dirac point to be $\omega_{D}^{\prime}=0.5325$ for \pm 25 modes in the RCWA transmittance calculations. Although the transmittance typically converges better than $1 \%$ with these numbers of modes, small shifts in the frequencies still occur. Using only \pm 15 modes instead of \pm 25 changes the optimal value for the Dirac frequency to 0.5318 , corresponding to a shift of $0.13 \%$. As a comparison, the difference in the value from the band-structure (0.5294) corresponds to a difference of $0.58 \%$. It is visible from Fig. 4 that the frequency must be determined precisely for large widths. A change in $\Delta \omega^{\prime}$ of 0.0002 can turn the $1^{\text {st }}$ channel from non-propagating to propagating, changing the scaling behavior significantly. In the band-structure, this would correspond to going away from the Dirac point into the conical region, where a larger range of $k_{11}$ is available.

Using the determined Dirac frequency, we calculate the length- and width-dependence of the transmittance (Fig.5 ). For a fixed width of $W=300$, we use a different number of channels corresponding to different $k_{\|, \max }$. Using more channels increases the transmittance for short crystals, but, since channels with a large $k_{\| 1}$ decay rapidly, the total transmittance becomes independent of this quantity after a length of approximately $1 / k_{1, \max } \cdot \underline{28}$ 

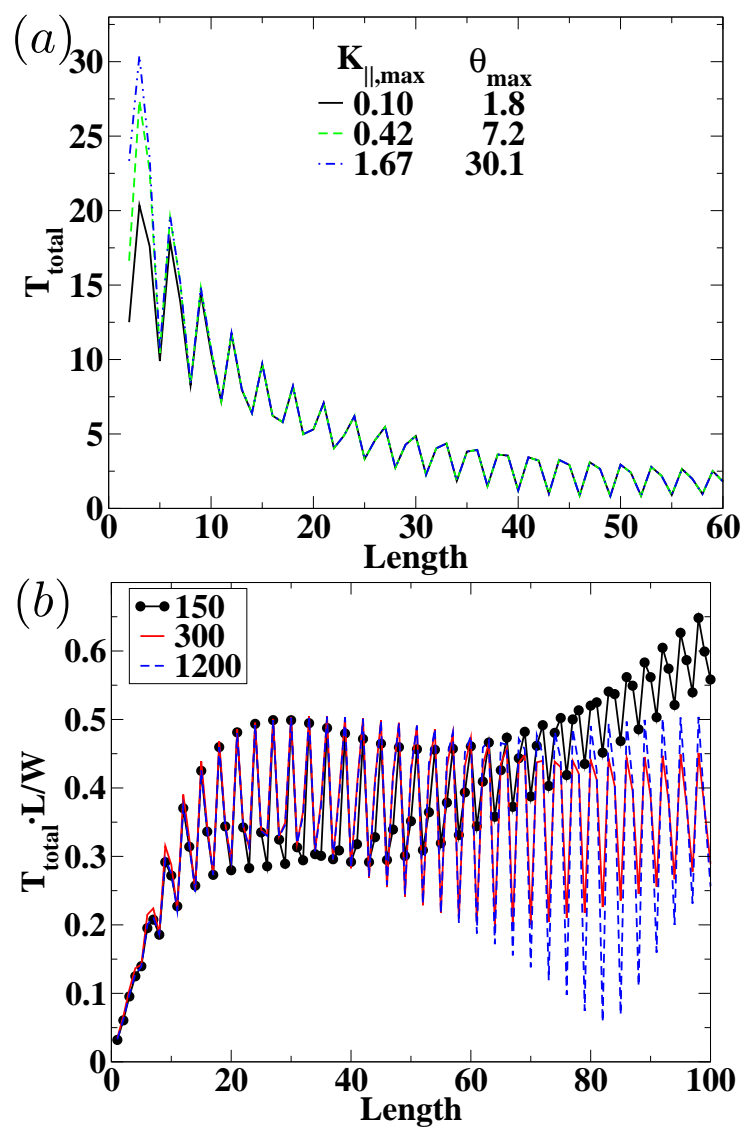

FIG. 5: (Color online) (a) Total transmittance $\sum_{n=-N}^{N} \mathrm{~T}_{k_{\|, n}}$ for $N=5,20$, and 80 with a fixed width of $\mathrm{W}=300$ corresponding to different $k_{\|, \max }$ and $\theta_{\max }$. The length, after which the results are equal in all cases, is determined by the smallest $k_{\|, \max }$. (b) Normalized transmittance TL/W. The oscillations are caused by the finite size of the structure. Different curves belong to different widths. The deviation of the black curve with the solid dots is due to the very narrow width. At approximately 40 unit cells, only the channel with $k_{\Perp, \max }=0$ contributes with a constant transmittance. Hence the increase in the rescaled transmittance.

According to the proposed scaling law, multiplying the total transmittance by a factor $\mathrm{L} / \mathrm{W}$ leads to a constant value.$^{28}$ Our results in Fig. 5 b are not constant but oscillate around a value of approximately 0.36 , slightly higher than the predicted value of $1 / \pi .^{28}$ The oscillations in the transmittance, which depend on the length and surface termination of the crystal, are Fabry-Perot resonances caused by the finite length. They do not exhibit a smooth curve since the sampling can only be completed in length steps of 1 unit cell and individual FabryPerot oscillations are not resolved. They can be resolved by fixing the length and varying the frequency in very small steps. Another deviation from the constant value can be seen 


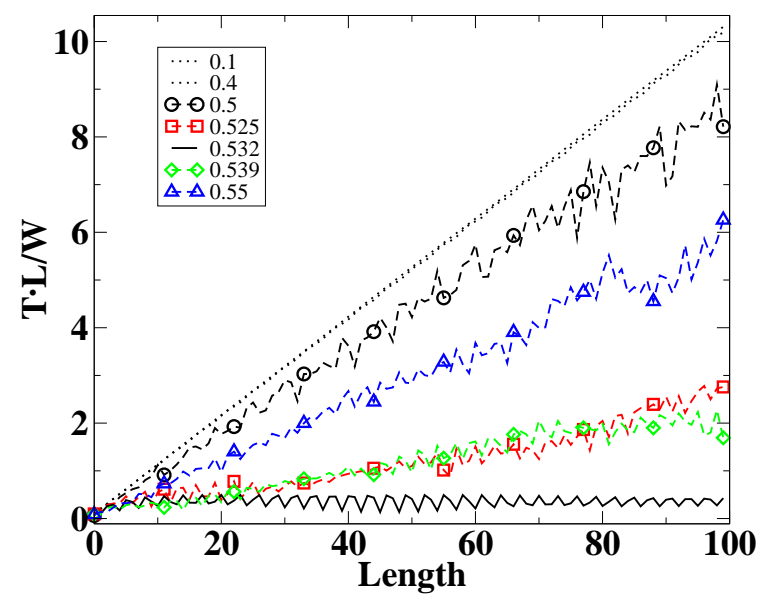

FIG. 6: (Color online) Rescaled transmittance as a fuction of length for different frequencies ( $N=10$, same $\theta_{\max }$ in all cases). We can distinguish 3 regimes in the plot. Far $(\omega=0.1)$ away from the Dirac point the rescaled transmittance increases linearly. Here, all channels contribute with a high transmittance $(\approx 1.0)$. Close to the Dirac point the slope is reduced, since only a fraction of the channels contributes. At the Dirac point $(\omega=0.532)$ the curve oscillates around 0.36 . The stop band region is not shown, but the curve would be close to zero for all lengths due to the exponential decay.

in the curve for a width of 150 unit cells (curve with solid circles). For this width, a linear increase in the normalized transmittance is visible, starting at a length of 40 unit cells. For structures longer than this width, only the $0^{\text {th }}$ channel contributes to transmittance with a constant value. The linear dependence of $\mathrm{TL} / \mathrm{W}$ is then caused by the multiplication of $\mathrm{T}$ with the length of the sample.

For different frequencies, we can identify several different characteristic behaviors for normalized transmittance (Fig.66). At low frequencies in the first band $(\omega=0.1)$, normalized transmittance is given by a straight line with the same slope for all frequencies, since all channels are contributing with a transmittance of about $100 \%$. The total transmittance $\mathrm{T}_{\text {total }}$ then corresponds to the number of channels. In the second band, the transmittance still grows linearly with different slopes, but oscillates around an average value. In this case, some of the channels are contributing and the total number of contributing channels determines the slope. At the Dirac point, a value around 0.36 is obtained as discussed before. The final regime is the stop band (not shown in the plot), where the normalized transmittance is always close to zero and decaying, since the effect due to the exponential decay is stronger 

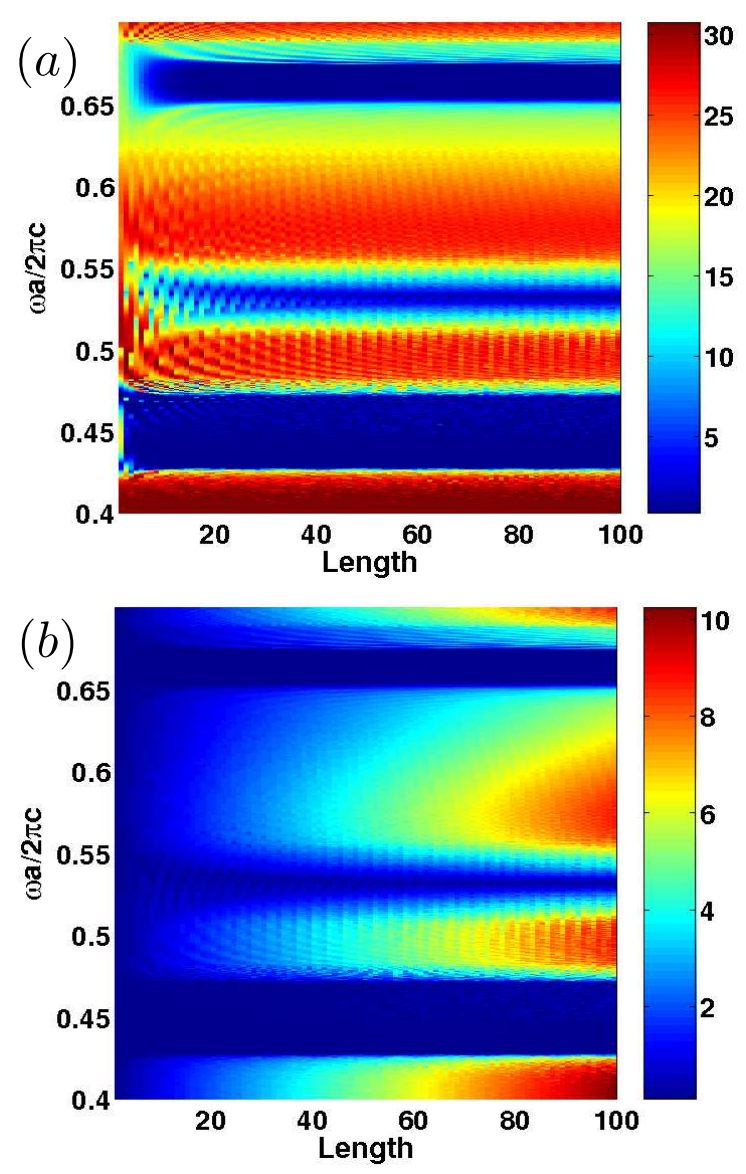

FIG. 7: (Color online) (a): Total transmittance $\mathrm{T}=\sum_{n} \mathrm{~T}_{k_{\|, n}}$ over length for different frequencies $(\mathrm{W}=600, \mathrm{~N}=15)$. The three dark regions are the lower stop band (0.45), the Dirac point (0.5325) and the quasi stop band (0.67). (b): Rescaled transmittance TL/W. In the stop band this value is approximately zero; whereas, at the Dirac point the value oscillates around 0.36.

than the increase caused by multiplication with the length. In all examples the maximum angle $\theta$ and the number of channels are fixed. The four different regimes are also visible in Fig. 8, which shows the total transmittance (a) and the normalized transmittance (b) for a wide range of frequencies over length.

A better understanding for the occurrence of these 4 regimes can be obtained by looking at the length-dependent transmittance of the individual channels for two example frequencies in Fig.8. Firstly, we consider a frequency close to the Dirac point (Fig.8a). For short crystals up to about 100 unit cells, the number of propagating channels decreases and for longer structures, nine channels contribute with a large transmittance. The rescaled transmittance is TL/W. Hence, it increases linearly for a length exceeding 100 unit cells. At the Dirac 

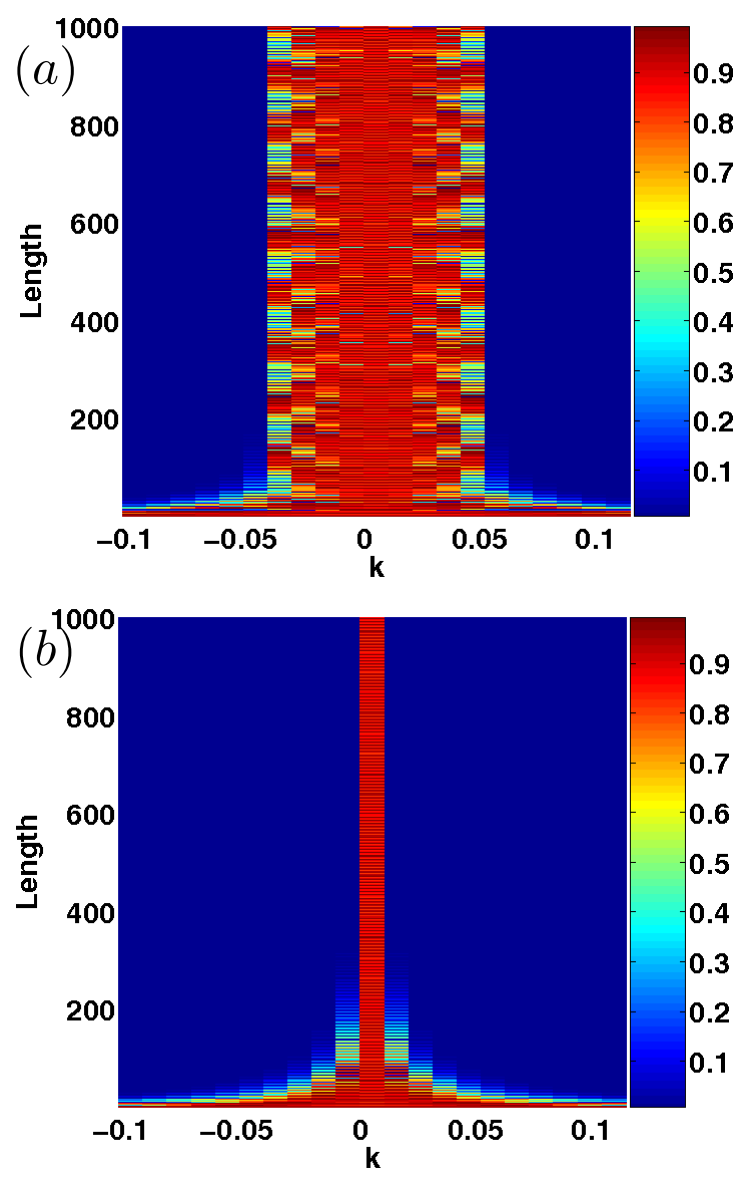

FIG. 8: (Color online) Length-dependence of the first \pm 10 channels for different frequencies. (a) $\omega^{\prime}=0.528$ corresponds to a line with an intermediate slope in Fig.6. (b) $\omega^{\prime}=0.5325=\omega_{D}^{\prime}$ belongs to the lowest line in Fig.6. In both cases the length scale is 10 times longer than in the previous graphs. The rescaled transmittance for lengths after which the number of propagating channels stays constant (approx. 100 (250) in a (b)) exhibits the same behavior as the curve with solid dots in Fig.5b at large lengths.

point (Fig.86b), all but the $0^{\text {th }}$ channel are suppressed for long structures. In the region up to a length of about 250 unit cells, the suggested scaling behavior is observed. Again, for longer structures TL/W will increase linearly, due to the constant transmittance of the $0^{\text {th }}$ channel comparable to the black curve with solid dots in Fig.5b.

Previously, it has been stated that the scaling of the transmittance is valid for lengths larger than $1 / k_{\|, \max }$ in the limit of $W>>L$. As discussed before, there also exists an upper limit for the length for this scaling behavior, which has not been addressed in previous publications. We determined this long length limit by comparing the $\pm 1^{\text {st }}$ channel to the 


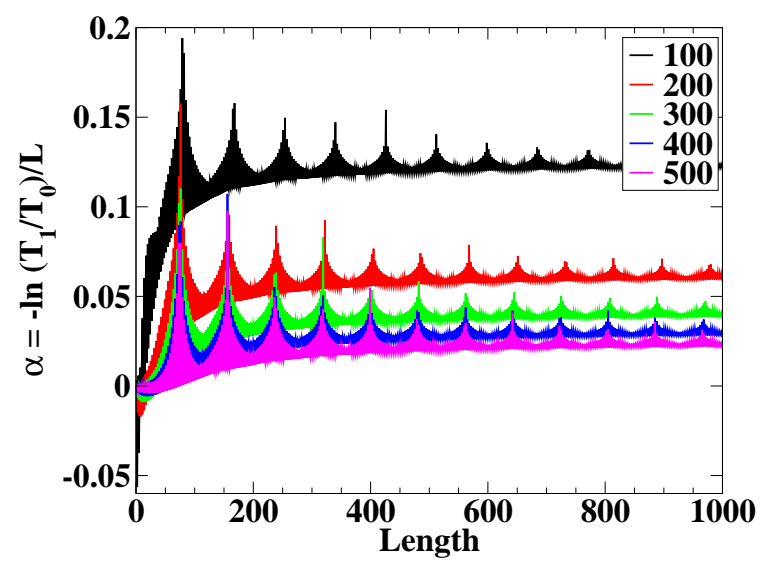

FIG. 9: (Color online) Damping constant $\alpha=-\ln \left(\mathrm{T}_{1} / \mathrm{T}_{0}\right) / L$ of the $1^{\text {st }}$ channel with respect to the $0^{\text {th }}$ channel for different widths. The highest curve corresponds to the structure with the smallest width. The resonances are due to the finite length of the structure.

$0^{\text {th }}$ one. All channels with $k_{\|} \neq 0.0$ decay exponentially at the Dirac point, since there are no propagating states available in the band-structure similar to the case in the gap. The propagating channels always contribute with a transmittance of approximate unity, so ignoring the details of the transmittance caused by the Fabry-Perot oscillations, we can express the total transmittance of all channels by

$$
\mathrm{T}_{\text {total }}=\mathrm{T}_{0}+\mathrm{T}_{1}+\mathrm{T}_{2}+\cdots=\mathrm{T}+\mathrm{e}^{-\alpha_{1} L} \mathrm{~T}+\mathrm{e}^{-\alpha_{2} L} \mathrm{~T}+\cdots
$$

with $\mathrm{T}$ on the order of 1 . Since the $2^{\text {nd }}$ and higher channels do not contribute significantly, we can define the relative damping of the $1^{\text {st }}$ channel with respect to the $0^{\text {th }}$ as $\alpha=-\ln \left(\mathrm{T}_{1} / \mathrm{T}_{0}\right) / L$, plotted for different widths in Fig.9. As long as the transmittance in the $1^{\text {st }}$ channel decays, the damping constant increases until it saturates and becomes constant.

To determine the maximum length for the $1 / \mathrm{L}$ scaling of the transmittance, the inverse of the damping constant, given by the length for a suppression is $1 / e$, can be used as a quantitative measure. This damping length is obtained by averaging the (length-dependent) damping constant, once it has reached a constant value and then inverting the average. The averaging procedure is required to reduce the impact of the Fabry-Perot resonances.

The results of this averaging are shown in Fig.10 and exhibit a linear behavior. For crystals with a very wide width, the saturated value is only reached for very long lengths. Not using sufficiently long structures leads to a damping constant, which is still increasing; 


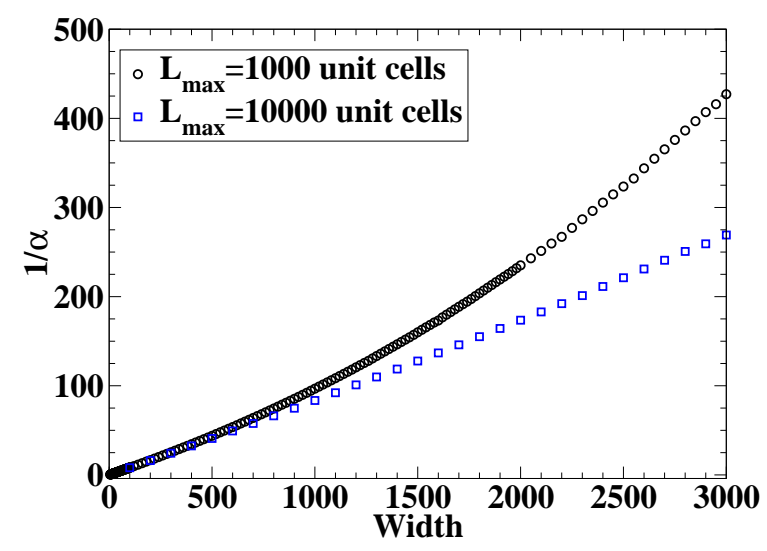

FIG. 10: (Color online) Damping length $1 / \alpha$ obtained by averaging the damping constant on the left and inverting. If the crystal is not sufficiently long the damping constant does not reach a constant value for very wide structures and the damping length deviates from linear behavior (circles $L_{\max }=1000$, squares $L_{\max }=10000$ ).

hence, to an overestimation of the damping length and a deviation from the linear behavior. From a linear fit, the slope can be obtained as 0.095, which gives a width to length ratio of about 10:1, meaning the length limit of the W/L scaling of the transmittance is reached at about $1 / 10$ of the width. Identification of the upper limit is important, if one wants to study disordered systems. In this case, the Dirac point may be shifted locally; hence, propagating modes are available in regions where no modes were available before. Consequently, this can lead to an enhanced transmittance in the vicinity of the Dirac point. If the channels with $k_{\|} \neq 0.0$ are suppressed less compared to $0^{\text {th }}$ channel in the disordered case than in the unperturbed structure, this will lead to a significant change in the damping length, even if all channels experience changed due to disorder. Studying these quantities allows a better understanding of the transmittance around the Dirac point in the case of disorder and offers the possibility to discuss the open question whether disorder will increase or decrease the transmittance in this region. ${ }^{28}$

In conclusion, we have presented detailed numerical calculations of the transmittance in hexagonal two-dimensional Photonic Crystals close to the Dirac point. We found the transmittance at the Dirac point is inversely proportional to the thickness of the sample. A detailed dependence of this behavior on the individual channels is given. We give an explanation and a criterion for an upper length limit of this behavior and relate it to the width of the crystal. The dependence of the transmittance away from the Dirac point is 
also examined. It was determined that the transmittance decays exponentially as expected when the frequency lies in the gap. When the frequency lies in the band, not only the $k_{\| 1}=0$ component is contributing to the transmittance for all lengths. The number of contributing channels depends on the width and the distance from the Dirac point frequency.

\section{Acknowledgments}

M.D. gratefully acknowledges financial support from the Alexander-von-Humboldt Foundation (Feodor-Lynen Program). Work at Ames Laboratory was supported by the Department of Energy (Basic Energy Sciences) under contract No. DE-AC02-07CH11358. This work was partially supported by the office of Naval Research (Award No. N00014-07-1-0359)

* diem@ameslab.gov

1 L. Martinez, A. Garcia-Martin, and P. Postigo, Opt. Express 12, 5684 (2004).

2 D. Cassagne, C. Jouanin, and D. Bertho, Phys. Rev. B 52, R2217 (1995).

3 D. Cassagne, C. Jouanin, and D. Bertho, Phys. Rev. B 53, 7134 (1996).

4 M. Plihal and A. A. Maradudin, Phys. Rev. B 44, 8565 (1991).

5 D. Cassagne, C. Jouanin, and D. Bertho, Appl. Phys. Lett. 70, 289 (1997).

6 F. Gadot, A. Chelnokov, A. D. Lustrac, P. Crozat, J.-M. Lourtioz, D. Cassagne, and C. Jouanin, Appl. Phys. Lett. 71, 1780 (1997).

7 C. M. Soukoulis, ed., Photonic Crystals and Light Localization in the 21st Century, vol. 563 of NATO Science Series C (Kluwer Academic, Dordrecht, The Netherlands, 2001).

8 H.-Y. Ryu, J.-K. Hwang, and Y.-H. Lee, Phys. Rev. B 59, 5463 (1999).

9 M. A. Kaliteevski, J. M. Martinez, D. Cassagne, and J. P. Albert, Phys. Rev. B 66, 113101 (2002).

10 E. Lidorikis, M. M. Sigalas, E. N. Economou, and C. M. Soukoulis, Phys. Rev. B 61, 13458 (2000).

11 D. Gerace and L. C. Andreani, Opt. Lett. 29, 1897 (2004).

12 G. von Freymann, W. Koch, D. C. Meisel, M. Wegener, M. Diem, A. Garcia-Martin, S. Pereira, K. Busch, J. Schilling, R. B. Wehrspohn, et al., Appl. Phys. Lett. 83, 614 (2003). 
13 M. Notomi, Phys. Rev. B 62, 10696 (2000).

14 S. Foteinopoulou and C. M. Soukoulis, Phys. Rev. B 67, 235107 (2003).

15 A. Berrier, M. Mulot, M. Swillo, M. Qiu, L. Thylén, A. Talneau, and S. Anand, Phys. Rev. Lett. 93, 073902 (2004).

16 K. Guven, K. Aydin, K. B. Alici, C. M. Soukoulis, and E. Ozbay, Phys. Rev. B 70, 205125 (2004).

17 P. V. Parimi, W. T. Lu, P. Vodo, J. Sokoloff, J. S. Derov, and S. Sridhar, Phys. Rev. Lett. 92, 127401 (2004).

18 R. Moussa, S. Foteinopoulou, L. Zhang, G. Tuttle, K. Guven, E. Ozbay, and C. M. Soukoulis, Phys. Rev. B 71, 085106 (2005).

19 R. Gajić, R. Meisels, F. Kuchar, and K. Hingerl, Phys. Rev. B 73, 165310 (2006).

20 K. Busch, G. von Freymann, S. Linden, S. Mingaleev, L. Tkeshelashvili, and M. Wegener, Physics Reports 444, 101 (2007).

21 S. Raghu and F. D. M. Haldane (2006), arXiv:cond-mat/0602501.

22 F. D. M. Haldane and S. Raghu (2005), arXiv:cond-mat/0503588.

23 A. K. Geim and K. S. Novoselov, Nature Materials 6, 183 (2007).

24 M. I. Katsnelson, Materials Today 10, 20 (2007).

25 A. Rycerz, J. Tworzydlo, and C. W. J. Beenakker, Europhys. Lett. 79, 57003 (2007).

26 P. M. Ostrovsky, I. V. Gornyi, and A. D. Mirlin, Phys. Rev. Lett. 98, 256801 (2007).

27 J. Tworzydlo, B. Trauzettel, M. Titov, A. Rycerz, and C. W. J. Beenakker, Phys. Rev. Lett. 96, 246802 (2006).

28 R. A. Sepkhanov, Y. B. Bazaliy, and C. W. J. Beenakker, Phys. Rev. A 75, 063813 (2007).

29 M. I. Katsnelson, Eur. Phys. J. B 51, 157 (2006).

30 X. Zhang (2007), arXiv.org/0710.0682.

31 R. A. Sepkhanov and C. W. J. Beenakker (2007), arXiv.org/0712.1158v2.

32 G. G. Moharam and T. K. Gaylord, J. Opt. Soc. Am. 71, 811 (1981).

33 D. M. Whittaker and I. S. Culshaw, Phys. Rev. B 60, 2610 (1999).

34 L. Li, J. Opt. Soc. Am. A (1996).

35 L. Li, J. Opt. Soc. Am. A 14, 2758 (1997).

36 L. Sheng and S. He, J. Opt. Soc. Am. A 19, 1021 (2002). 